\title{
The ultra-low emittance synchrotron storage rings: a new paradigm for matter characterization.
}

\author{
Jean Susini \\ Synchrotron SOLEIL, Gif sur Yvette, France; \\ jean.susini@synchrotron-soleil.fr
}

Over the last few years, photon science community has been experiencing a revolution with the advent of ultra-low emittance storage rings based on Multi-Bend Achromat (MBA). In addition to green fields projects MAXIV (1), SIRIUS (2), and HEPS (3) in operation, commissioning, or construction, respectively, many third-generation facilities undertook major upgrades such as ESRF-EBS (4), APSU (5), ALS-U (6), SLS-2 (7), DLS-2 (8), etc. All are aiming to achieve unparalleled performances in terms of average spectral brilliance, coherent flux, and nano-focusing capabilities.

After an introduction of the main concepts behind this new revolutionary concept, the new characterization techniques and their potential for new applications will be discussed, for two distinct examples, including the commissioning and operation of the ESRFEBS $(6 \mathrm{GeV})$ and the project SOLEIL $(2.75 \mathrm{GeV})$ upgrade:

Since 2015 the ESRF has prepared the replacement of its old storage ring based on the double-bend achromat lattice by the EBS storage ring(9) based on the newly developed HMBA lattice with seven bending magnets per cell. During a long shutdown the EBS storage ring was installed in 2019 and went into its commissioning phase in December 2019. The EBS storage ring was successfully commissioned as the first fourth generation high energy synchrotron light source during the first six month in 2020. Nominal beam parameters could be confirmed early on in the process and the beamlines resumed user operation in September 2020 as planned. The expected improvement of the key beam parameters in terms brilliance, coherence and flux were confirmed across the entire beamline portfolio. Details on the commissioning of the beamlines and the performance reached will be presented together with early scientific results.

In 2019, SOLEIL launched a CDR (10) for an upgrade of its 20 years old storage ring with the ambition to produce round electron beams with a record low emittance of less than $50 \mathrm{pm} . r a d \times 50 \mathrm{pm} . r$ ad, hence photon beams with an exceptional brilliance exceeding by two orders of magnitude the performances of the current source. The very broad spectral range of Soleil from $\mathrm{THz}$ to tens of $\mathrm{KeV}$ is a challenge but offers unique scientific opportunities which will be discussed and illustrated by examples in materials science and biology.

[1] Tavares, P. F., et al., "Status of the MAX-IV Accelerators", IPAC 2019 proceedings, TUYPLM3, 1185-1190 (2019).

[2] Liu, L., "SIRIUS Commissioning Results", IPAC 2020 (2020).

[3] Jiao Y., "The HEPS Project”, Journal of Synchrotron Radiation, 25, 1611-1618 (2018).

[4] Raimondi P., "Hybrid Multi Bend Achromat: from SuperB to EBS", 8th International Particle Accelerator Conference, May 2017, Copenhagen, Denmark, 3670-3675,10.18429/JACoW-IPAC2017-THPPA (2017).

[5] Borland, M. et al., "The Upgrade of the Advanced Photon Source”, IPAC 2018 proceedings, THXGBD1, 2872-2877 (2018).

[6] Steier, C. et al., "Design Progress of ALS-U, the Soft X-Ray Diffraction Limited Upgrade of the Advanced Light Source, IPAC 2019 proceedings, 1639-1641 (2019).

[7] Streun, A., et al., "SLS-2: the Upgrade of the Swiss Light Source", Journal of Synchrotron Radiation, 25, 631-641 (2018).

[8] https://www.diamond.ac.uk/Home/About/Vision/Diamond-II.html

[9] Orange Book: http://www.esrf.eu/home/orange-book.html

[10] CDR SOLEIL, https://www.synchrotron-soleil.fr, to be published

Keywords: DLSR, Synchrotron Radiation, coherence 